\title{
PET-CT Scan Without Contrast
}

National Cancer Institute

\section{Source}

National Cancer Institute. PET-CT Scan Without Contrast. NCI Thesaurus. Code C142342.

A form of PET/CT imaging in which radiopharmaceuticals is not used. 